\title{
Number Systems from a General Point of View
}

\author{
Milosav M. Marjanović \\ Serbian Academy of Sciences and Arts, Belgrade, Serbia \\ Email: milomar@beotel.net
}

How to cite this paper: Marjanović, M.M. (2019) Number Systems from a General Point of View. Open Access Library Journal, 6: e5484.

https://doi.org/10.4236/oalib.1105484

Received: May 20, 2019

Accepted: June 29, 2019

Published: July 2, 2019

Copyright ( 2019 by author(s) and Open Access Library Inc.

This work is licensed under the Creative Commons Attribution International License (CC BY 4.0).

http://creativecommons.org/licenses/by/4.0/

Open Access

\begin{abstract}
This paper presents recent results of this author and Z. Kadelburg and also contains additional comments and remarks. These results complete the study of number systems which this author has published in OALib Journal in search of a ground for designing a simplified content assigned to primary teachers. This ground consisted of derivation of properties of the system $N$ of natural numbers with 0 and of the use of these properties as a basis for the extension of $N$ to the systems of positive rational numbers and the system of integers. The crucial step in our final research paper has been the selection of basic operative properties of the system $N$ (properties of operations and the order relation). Using these properties and those deduced from them, the system $Q_{+}$of positive rational numbers with 0 has been constructed and its basic operative properties verified (being those of $N$, plus the property of existence of multiplicative inverse). Then, using properties of $Q_{+}$, the system $Q$ of rational numbers is constructed and its properties verified (being those of $Q_{+}$, plus the existence of additive inverse). Taking the basic operative properties of $N$ for the axioms, we define the algebraic structure $\{S,+, \times,<\}$, where $S$ is a non-empty set, " + " and " $x$ " two binary operations and " $<$ " the order relation, which we call $N$-structure, The number systems $N, Q_{+}$and $Q$, as well as the system $R$ of real numbers are examples of $N$-structure. Thus, they share all properties that the axioms express and all those deduced from them. Hence all these properties when transcribed with the letters denoting the corresponding variables are transferable from $N$ to the systems $Q_{+}, Q$ and $R$. This is a precise formulation of the Peacock's principle of permanence and a firm logical basis for its validity. Finally, we prove that each system satisfying the axioms of $N$-structure contains an isomorphicb copy of $N$. Thus, the natural numbers with 0 are the smallest system satisfying the axioms of $N$-structure, This is a characterization of the system $N$ which is analogous to that of $Q$ which is the smallest ordered field.
\end{abstract}

\section{Subject Areas}

Education 


\section{Keywords}

Number Systems, Logical Foundation for Peacock's Principle of Permanence, Characterization of the System of Natural Numbers

\section{Introduction}

At the start of this paper, we begin with making a few brief observations on the historical development of the idea of real number and, at the same time, these observations will be followed by the references on teaching mathematics.

Just having a glance at the Euclid's Elements of Geometry (see [1]), it is immediately seen that by far the most elaborate theme of this classical piece of work is the theory of (positive) real numbers (expressing it in the contemporary language of mathematics). Out of thirteen books of the Elements, six of them: books 5, 7, 8, 9, 10 and 12 are primarily concerned with the Eudoxus conception of (positive) real numbers, their properties expressed in terms of proportions and their applications.

Although unified by the idea of real number as being ratio of magnitudes of the same kind, the intuitively much more approachable ideas of natural numbers and their ratios (rational numbers) are separately considered in the Elements. Thus the development of number idea starts with natural numbers, goes via rational numbers and terminates with real numbers, what is a path followed from antiquity to the modern time.

A significant simplification of the Eudoxus theory happened when Descartes invented the number axis-a geometric model where the conveyors of the meaning of real numbers are straight line segments having an end the origin and where arithmetic operations are feasible as simple geometric constructions (multiplication and division as construction of the fourth proportional). This invention has had a powerful effect on the further development of the idea of real number and has entered school curricula as an inevitable device for the elaboration of the didactical theme-real numbers.

Everybody can conjure up his/her own memories from school days and evoke the long period of at least four years of learning natural numbers, two years of learning rational numbers (usually interpreted as fractions) and, at the end, a number of lessons devoted to irrational numbers. Some examples of irrational numbers are given together with the proofs that they are not rational ( $\sqrt{2}$ being a typical example), $\pi$ is also included with the remark that the proof of that fact is far beyond the scope of school mathematics. The fact that repeating decimals represent rational numbers and non-repeating irrational is also a ground for a number of corresponding examples but the most relaxing are the words of the teachers when they say that "when we calculate, we use irrational numbers as they were rational". This is enough to convince the 14-year-old students of the existence of numbers which are not rational and to supply with meaning the no- 
tations of some constants that figure in geometric formulas.

In calculus, with its concepts of continuous function and limit, the continuum of real numbers is needed for its rigorous foundation. Richard Dedekind in his essay Continuity and Irrational Numbers (German original published in 1872, an English translation can be found in [2]) uses the partitions of the set of rational numbers, to which we today refer as Dedekind cuts, to define rational and irrational numbers to fit together forming so the real number continuum. This continuum is a foundation stone of the infinitesimal analysis and is also the introductory teaching theme of the courses which embrace this mathematical content. For young university students, Dedekind cuts are certainly unusual conveyors of the meaning of numbers and every lecturer who ever tried to teach real numbers this way must remember apathetic faces of his/her students. Today, majority of lecturers prefer to take the real number continuum to be the continuous ordered field and are concentrated predominantly on continuity axiom and its applications: properties of continuous real valued functions, existence of elementary functions, proofs of existence of some limits, etc.

\section{My Personal Refreshing of the Interest for Number Systems}

In early 1960's I began to teach a two-year omnibus course of calculus for students of physics and later on I had to teach a two-year course of infinitesimal analysis for students of mathematics. Since that time on, a silent longing for an overture to such courses which would combine student's knowledge and skill gained through learning mathematics in school with a deeper understanding of number systems, has always existed in my mind.

My interest was refreshed when, a couple of years ago, I participated in a discussion about which contents should be included in the math courses for students that are trained to be primary teachers. My stand was that, first of all, these students should be acquainted, at least up to some degree and possibly not very formally, with the development of number systems. As for math students, my firm opinion is that their didactics of mathematics courses should thoroughly present this development with all essential historic references.

As the result of my renewed interest for number systems and thanks to the freedom to think and research that OALib Journal provides for the authors, I have published my papers [3] and [4]. In these papers algebra is used when a series of properties of proportions and equalities that relate differences are established in the system of natural numbers. Then, this system is extended by ratios $m: n,(n>0)$ of natural numbers and, in that way, the system of positive rational numbers with 0 is constructed. The properties of this extended system are verified relying upon the properties of natural numbers. Extending the system of natural numbers by formal differences $m-n$, the system of integers is constructed and its properties are also verified dependently on the properties of natural numbers. 
When these extensions have been realized, we were governed by the Peacock's principle of permanence of equivalent forms: "Whatever algebraical forms are equivalent, when the symbols are general in form but specific in value, will be equivalent likewise when the symbols are general in value as well as in form." This is a very suggestive principle but it lacks precision and does not tell clearly which properties of natural numbers can be carried over to the extended systems. Some vague ideas that $I$ had at the end were not clear enough to continue this research. But with time, these ideas have been cleared up and the authors of the paper [5] have shaped them in the desired form. This paper, together with this author's previous papers [3] and [4], make a complete package and I feel obliged to present it to the interested readers of OALib Journal, together with some additional comments.

\section{Basic Operative Properties of the System $N$ of Natural Numbers}

Now we present the content of the paper [5], suggesting to the reader to also read it directly.

When the set $N$ of natural numbers is viewed as the structure $\{N,+, \times,<\}$, consisted of the set $N$, the addition + and the multiplication $\times$, together with the order relation <, we have to add the basic properties of these operations and the order relation, hoping that such properties (together with those deduced from them) will suffice as a ground for extension of this system. Selection of these properties that we call basic operative properties of the system $N$ with 0 , has been a crucial step and they are listed right below:

1) $(\forall k)(\forall l) k+l=l+k$

2) $(\forall k)(\forall l)(\forall m)(k+l)+m=k+(l+m)$

3) $(\exists 0)(\forall k) k+0=k$

4) $(\forall k)(\forall l) k l=l k$

5) $(\forall k)(\forall l)(\forall m)(k l) m=k(l m)$

6) $(\exists 1)(0<1$ and $(\forall k) k \times 1=k)$

7) $(\forall k)(\forall l)(\forall m) k(l+m)=k l+k m$

8) $(\forall k)(\forall l)(k<l \Leftrightarrow(\exists m>0) k+m=l)$

9) $(\forall k)(\forall l)(k<l$ or $k=l$ or $l<k)$

10) $(\forall k)(\forall l)(\forall m)(k<l \Leftrightarrow k+m<l+m)$

11) $(\forall k)(\forall l)(\forall m>0)(k<l \Leftrightarrow k m<l m)$

\section{List 1}

(To denote variables in $N$ we use letters: $i, j, k, l, m, n, \cdots$ )

As usual, two "partial" operations are defined on $N$, in the following way:

1) When $k+l=m$, then $l$ is called the difference of $m$ and $k$ and is denoted by $m-k$.

2) When $k l=m$ and $k \neq 0$, then $l$ is called the quotient of $m$ and $k$ and is denoted by $m: k$. 
Using the basic operative properties of $N$, a long series of deduced properties have been listed, predominantly being the properties of proportions and the equalities relating differences, which are planned to be used for extension of $N$.

\section{Extensions of the System $N$}

Extending the system $N$ by the set of all ratios $k: l$ where $k$ and $l>0$ are arbitrary natural numbers, the equivalence relation is defined in this set, when two ratios $k: l$ and $m: n$ are taken to be equivalent when $k n=l m$. (The motivation for this definition is the property of $N$ : Two quotients $k: l$ and $m: n$ are equal if $k n=l m$ ). The set of these equivalence classes is called the set of positive rational numbers with 0 and is denoted by $Q_{+}$. For the sake of simplicity, the equivalence classes $[k: l]$ are denoted by $k: l$. The operations of addition and multiplication and the order relation on $Q_{+}$are defined, taking

$$
\begin{gathered}
(k: l)+(m: n)=(k n+l m):(l n) \\
(k: l) \times(m: n)=(k m):(l n) \\
(k: l)<(m: n) \Leftrightarrow k n<l m
\end{gathered}
$$

(The motivation for these definitions is formally the same set of relations valid in $N$, when these ratios are quotients defined in that set). These definitions are taken to be correct after the verification that they are independent of the particular choice of the representatives of equivalence classes. Proceeding further, a verification also shows that all properties on List 1 continue to be true in $Q_{+}$, adding a new one: For each positive rational number $k: l,(k>0)$, the number $l: k$ is also positive and $(k: l) \times(l: k)=1: 1=1$. For $k: l, l: k$ is called the multiplicative inverse and is denoted by $(k: l)^{-1}$.

In order to compare more clearly basic properties of the systems $N$ and $Q_{+}$, we use letters $q, r, s, t, \cdots$ to denote variables in $Q_{+}$and we list the basic properties of this system right below:

1) $(\forall q)(\forall r) q+r=r+q$

2) $(\forall q)(\forall r)(\forall s)(q+r)+s=q+(r+s)$

3) $(\nexists 0)(\forall q) q+0=q$

4) $(\forall q)(\forall r) q r=r q$

5) $(\forall q)(\forall r)(\forall s)(q r) s=q(r s)$

6) $(\exists 1)(0<1$ and $(\forall q) q \times 1=q)$

7) $(\forall q \neq 0)(\exists r) q r=1$

8) $(\forall q)(\forall r)(\forall s) q(r+s)=q r+q s$

9) $(\forall q)(\forall r)(q<r \Leftrightarrow(\exists s>0) q+s=r)$

10) $(\forall q)(\forall r)(q<r$ or $q=r$ or $r<q)$

11) $(\forall q)(\forall r)(\forall s)(q<r \Leftrightarrow q+s<r+s)$

12) $(\forall q)(\forall r)(\forall s>0)(q<r \Leftrightarrow q s<r s)$

List 2 
Omitting property 7) from List 2, then remaining properties are formally identical with the properties on List 1 , as well as are identical all properties that can be deduced from them. Only the letters denoting variables are different. But what is essentially different are the elements of sets that these variables denote: $k, l, m, n, \cdots$ elements of $N$ and $q, r, s, t, \cdots$ elements of $Q_{+}$. For example, in [5], the following property of $N$ was deduced: (12) $(k: l):(m: n)=(k n):(l m)$. Transcribing it into the property of $Q_{+}$, we get the relation: (12') $(q: r):(s: t)=(q t):(r s)$ (and there is no need for verification of its validity). Paraphrasing Peacock in his own words, we have: $(k: l):(m: n)=(k n):(\operatorname{lm})$ is general in form but specific in value while $(q: r):(s: t)=(q t):(r s)$ is general in value as well as in form.

Here and in this way, we demonstrate precisely that the basic properties of $N$ together with all those deduced from them are transferable to $Q_{+}$and we have shown that this transfer has its clear logical justification.

The system $Q_{+}$is extended using the set of formal differences $q-r$ and defining the equivalence relation on this set, taking $q-r \approx s-t$ if $q+t=r+s$. The set of these equivalence classes is called the set of rational numbers and is denoted by $Q$. We denote the equivalence class $[q-r]$ by any of its representatives $q-r$ and we define the operations of addition and multiplication as well as the order relation, relying on the relations deduced in $N$ and then transcribed in $Q_{+}$:

$$
\begin{gathered}
(q-r)+(s-t)=(q+s)-(r+t) \\
(q-r) \times(s-t)=(q s+r t)-(q t+r s) \\
q-r<s-t \Leftrightarrow q+t<r+s
\end{gathered}
$$

The correctness of these definitions is checked, verifying that their meaning does not depend on specific choice of representatives.

Further on, all basic properties from List 2 are transcribed and their validity is checked. Being $(q-r)+(r-q)=(q+r)-(r+q)=(0-0)$, it follows that each $(q-r)$ has the additive inverse $(r-q)$. Adding this new property to the list of transcribed properties, the following list of the basic operative properties of $Q$ is obtained:
1) $(\forall a)(\forall b) a+b=b+a$
2) $(\forall a)(\forall b)(\forall c)(a+b)+c=a+(b+c)$
3) $(\exists 0)(\forall a) a+0=a$
4) $(\forall a)(\exists b) a+b=0$
5) $(\forall a)(\forall b) a b=b a$
6) $(\forall a)(\forall b)(\forall c)(a b) c=a(b c)$
7) $(\exists 1)(0<1$ and $(\forall a) a \times 1=a)$
8) $(\forall a \neq 0)(\exists b) a b=1$
9) $(\forall a)(\forall b)(\forall c) a(b+c)=a b+a c$ 
10) $(\forall a)(\forall b)(a<b \Leftrightarrow(\exists c>0) a+c=b)$

11) $(\forall a)(\forall b)(a<b$ or $a=b$ or $b<a)$

12) $(\forall a)(\forall b)(\forall c)(a<b \Leftrightarrow a+c<b+c)$

13) $(\forall a)(\forall b)(\forall c>0)(a<b \Leftrightarrow a c<b c)$

List 3

Omitting from List 3 two properties expressing the existence of inverse elements, the remaining properties are formally identical with those on the List 1 with which they also have the same set of deduced properties. Thus, we see that all operative properties (basic and deduced) of the system $N$ are transferable to the system $Q$ and this transfer has again a clear logical justification.

\section{A Wider View of Number Systems}

Let $\{S,+, \times,<\}$ be an ordered quadruple, where " $S$ " is a non-empty set, " + ' and " $X$ " are two binary operations and " $<$ " is an order relation. When the properties on List 1, List 2 or List 3 are taken to be axioms, this quadruple becomes an algebraic structure which we will call $N$-structure, $Q_{+}$-structure and $Q$-structure, respectively. We use now provisional terms for these structures and let us note that $Q$-structure is standardly called the ordered field. Each of these structures has as an example, a system whose properties satisfy the corresponding axioms: for $\mathrm{N}$-structure the system of natural numbers with 0 , for $Q_{+}$-structure the system of positive rational numbers with 0 and for $Q$-structure the system of rational numbers. Thus, the existence of these systems demonstrates that the axioms of the above structures are not contradictory. Let us notice that the existence of the systems $Q_{+}$and $Q$ is ensured by their construction and verification of their properties. As it is well known, the system $R$ of real numbers is characterized as the continuous ordered field but its existence is ensured by a direct construction (Dedekind cuts, Cauchy sequences, infinite decimal fractions) or fairly satisfactory, as it is done in school, by geometric interpretation on the number axis.

The axioms of $Q$-structure are more restrictive than those of $Q_{+}$-structure and the least restrictive are the axioms of $\mathrm{N}$-structure. Therefore, we see that the systems of rational and real numbers as well as the system of positive rational numbers with 0 are also the examples of $N$-structure. Thus, we conclude that the basic operational properties of the system of natural numbers together with those deduced from them are also the properties of positive rational, rational and real number systems, when they are transcribed writing the corresponding variables of each of these systems. This is a very precise and logically justified formulation of the Peacock's principle of permanence. Let us remark that the original formulation of the Peacock's principle is suggestive but it lacks the necessary precision and a firm logical ground for its validity. In that time the abstract ideas of set and structure did not exist yet and they were created some fifty years afterwards.

Now let us suppose that $\{S,+, \times,<\}$ is an example of $N$-structure. We define 
inductively a sequence $N^{\star}$ in $S$ starting with $a_{0}=0_{S}$ and $a_{1}=1_{S}$. Supposing that $a_{n}$ has been defined, we take $a_{n+1}=a_{n}+1_{S}$.

Let us prove that $a_{n+m}=a_{n}+a_{m}$. This relation is true when $m=1$ and let us suppose that $a_{n+m}=a_{n}+a_{m}$ (this is the inductive hypothesis). Then,

$a_{n+(m+1)}=a_{(n+m)+1}=a_{n+m}+1_{S}=\left(a_{n}+a_{m}\right)+1_{S}=a_{n}+\left(a_{m}+1_{S}\right)=a_{n}+a_{m+1}$, what was to be proved.

Let us also prove that $a_{n \times m}=a_{n} \times a_{m}$. For $m=1$, we have

$a_{n \times 1}=a_{n}=a_{n} \times 1_{S}=a_{n} \times a_{1}$. Supposing that $a_{n \times m}=a_{n} \times a_{m}$, we have

$a_{n(m+1)}=a_{n m+n}=a_{n m}+a_{n}=a_{n} \times a_{m}+a_{n} \times 1_{S}=a_{n}\left(a_{m}+1_{S}\right)=a_{n} \times a_{m+1}$.

Since for each $n, a_{n}<a_{n+1}$, the sequence $N^{\star}$ is increasing. Thus, $n<m$ implies $a_{n}<a_{m}$.

Taking all above facts into account, we see that the mapping $n \rightarrow a_{n}$ is an isomorphism and $N^{*}$ is an isomorphic copy of the system of natural numbers with 0 . Since the system $N$ is an example of $N$-structure and each example of $N$-structure contains, as a subsystem, a copy of the system $N$, we conclude that the system $N$ of natural numbers with 0 is the smallest system satisfying the axioms of the $N$-structure. This is a characterization of the system of natural numbers with 0 analogous to that one which characterizes the system of rational numbers as the smallest ordered field. (Though there exists a variety of different meanings of the term "semifield", $N$-structure should be called the "ordered semifield". Then, we can say that the system $N$ of natural numbers with 0 is the smallest ordered semifield).

We should add that H. Grassmann, in 1861, exploited a mapping similar to $n \rightarrow a_{n}$ when defining addition and multiplication in the set of natural numbers and when proving basic properties of these operations (see, for example [6]).

\section{Conclusion}

When the logical rigor is priority, the system of natural numbers starts $a b$ ovo-with the Peano axioms. When a deeper understanding of school arithmetic is in the first place, then its content is condensed and expressed in the terms of didactics of mathematics. Instead of being directly relied on set theory, the school arithmetic is seen based on set theoretical models which describe the teaching procedure. Thus, instead of the one-to-one correspondences and equipotent sets, Cantor principle of invariance of number expresses the natural dependence of the concept of number on the experience of collections of visible objects in the surrounding space as well as additive and multiplicative schemes model all those situations which convey the meaning related to addition and multiplication (see [3]). Further on, gradual building of the number blocks up to 10 , to 20 , to 100 , to 1000 and so on inductively produces for each natural number, a unique decimal notation, which is used when these numbers are compared. To look at and think about school arithmetic in this way it becomes consolidated and it forms a firm ground for derivation of basic operative properties of the 
system of natural numbers.

\section{Conflicts of Interest}

The author declares no conflicts of interest regarding the publication of this paper.

\section{References}

[1] Euclid's Elements of Geometry (Edited and Provided with a Modern English Translation by Richard Fitzpatrick).

[2] Dedekind, R. (1901) Theory of Numbers. The Open Court Publishing Company, Chicago.

[3] Marjanovic, M.M. (2017) A Survey of the System N of Natural Numbers Assigned to Primary Teachers. Open Access Library Journal, 4, 1-18. https://doi.org/10.4236/oalib.1103665

[4] Marjanovic, M.M. (2018) Extensions of the System N of Natural Numbers Assigned to Primary Teachers. Open Access Library Journal, 5, 1-11. https://doi.org/10.4236/oalib.1104096

[5] Marjanovic, M.M. and Kadelburg, Z. (2019) Structuring Systems of Natural, Positive Rational and Rational Numbers. The Teaching of Mathematics, XXII, 1-16.

[6] Dieudonné, J. (1987) Pour l'honneur de l'esprit humain, Hachette. 